\title{
Une analyse sémio-discursive de la culpabilisation des ménages modestes
}

La « crise de 2008 » expliquée par lemonde.fr, liberation.fr et lafinancepourtous.fr

Thierry Guilbert

\author{
(2) OpenEdition \\ Journals \\ Édition électronique \\ URL : http://journals.openedition.org/ei/5847 \\ DOI : 10.4000/ei.5847 \\ ISSN : 2553-1891 \\ Éditeur
}

Association Économie et Institutions

Référence électronique

Thierry Guilbert, "Une analyse sémio-discursive de la culpabilisation des ménages modestes », Économie et institutions [En ligne], 25 | 2017, mis en ligne le 20 décembre 2017, consulté le 19 avril 2019. URL : http://journals.openedition.org/ei/5847 ; DOI : 10.4000/ei.5847

Ce document a été généré automatiquement le 19 avril 2019

Revue Économie et institutions 


\section{Une analyse sémio-discursive de la culpabilisation des ménages modestes}

La « crise de 2008 » expliquée par lemonde.fr, liberation.fr et

lafinancepourtous.fr

Thierry Guilbert

1 La question traitée dans cet article concerne le « gouvernement de la crise » de 2008 dans les médias. Notre hypothèse est que, une forme de gouvernementalité a dû «gouverner dans/avec la crise » afin d'éviter un effondrement du système bancaire et financier, elle a également dû "gouverner la crise » à destination des citoyens. La forme transitive gouverner la crise ne relève pas de l'oxymore ${ }^{1}$, elle signifie pour nous gouverner la part symbolique (et donc discursive) de la crise, voire, comme le titrait la revue Lignes en 2009, utiliser « [...] la crise comme méthode de gouvernement ».

2 Les notions de gouvernement et de gouvernementalité étant associées au savoir/pouvoir de l'économie politique (Foucault 2001a), nous estimons que la "pédagogie de l'économie $»^{2}$ - dont l'« éducation financière » est l'un des avatars - joue aujourd'hui un rôle non négligeable dans le gouvernement des conduites, surtout lorsqu'elle est liée à un contexte de crise. Nous nous intéressons ici au rôle joué par les médias dans l'explication de la « crise de 2008 ", autrement dit à une forme de gouvernement de la crise.

La première partie de cet article présente notre cadre théorique, celui de l'analyse du discours, puis revient brièvement, d'une part, sur le concept de gouvernementalité en lien avec les notions de normalisation et de communication médiatique et, d'autre part, sur l'utilisation discursive du mot crise dans et par les médias.

4 La deuxième partie analyse notre corpus médiatique. Nous illustrerons le premier temps du gouvernement de la crise par l'étude du positionnement général «à chaud » de la presse écrite française dans deux journaux en ligne: libération.fr et lemonde.fr du 16 au 19 septembre 2008. Le site pédagogique lafinancepourtous.fr de l'Institut pour l'Éducation Financière du Public (IEFP) et le dossier «Crise de 2008 » publié le 22 octobre 2008, puis 
revu le 7 février 2013, nous permettrons ensuite d'analyser le discours «à froid» d'un autre type de média. Il s'agit d'analyser comment la crise y est (re)présentée à travers le lexique, les métaphores, les procédés discursifs et iconographiques utilisés, autrement selon une perspective à la fois discursive et sémiologique.

\section{L'analyse du discours, la gouvernementalité et la communication médiatique}

5 Nos travaux s'inscrivent en Analyse du discours (AD), champ des sciences du langage qui s'inspire des travaux de M. Foucault (2001b [1966], 1971) et de L. Althusser (1976 [1970]). L'AD considère que la signification d'un énoncé ne peut être saisie que dans son contexte de production, lequel comprend aussi bien les protagonistes que les conditions spatiotemporelles et sociohistoriques. Un discours n'est ni le reflet fidèle des choses, ni une transmission transparente d'information, mais véhicule et constitue des représentations de la réalité. Il s'ensuit que les discours traversent et façonnent les sujets parlants, les situations et les institutions. Les discours sont donc dans une relation d'intrication avec les institutions dont ils sont le produit en même temps qu'ils les font exister (Maingueneau 1995). Dès lors, il est possible d'avancer que les discours ont des effets sur les savoirs (Pêcheux 1969), qu'ils exercent un pouvoir, voire, comme le considère la Critical Discourse Analysis, qu'ils sont l'un des principaux instruments du pouvoir (Fairclough 1995).

\subsection{La gouvernementalité des médias et la normalisation}

6 C'est dans ce cadre théorique rapidement brossé que nous considérons la notion de gouvernementalité. Foucault (2004 [1977-1978]) inscrit celle-ci dans une "méthode historique de type généalogique » (Gautier 2007, p. 20, soul. par l'auteur) ou encore dans une "enquête généalogique de l'État », c'est-à-dire dans "l'examen des modalités par lesquelles s'exerce cette forme spécifique de pouvoir qui a pour cible la population » (Malette 2006, p. 58). Il ne s'agit pas pour Foucault d'étudier ce qu'est le pouvoir mais d'analyser les «mécanismes de pouvoir» (Foucault 2004, p.3). Les modalités ou « pratiques effectives » (Gautier 2007, p. 23) par lesquelles il s'exerce dessinent justement une forme de gouvernement, une gouvernementalité.

7 Le terme "gouvernement» chez Foucault rend compte, selon Malette, de deux acceptions :

D'une part, il réfère à une "conduite des conduites", c'est-à-dire à ces comportements qui ont pour objectif de modifier, guider ou influencer le comportement d'un groupe ou d'un individu. D'autre part, il réfère aux activités que nous associons à une forme d'entité décisionnelle à la tête de ce que nous nommons généralement l'“État". Lorsqu'elle désigne cette “conduite des conduites", cette activité de "gouverner" peut se comprendre de plusieurs façons. Elle peut en effet s'appliquer autant à une relation entre l'individu et lui-même ; à une relation interpersonnelle impliquant une forme de guidance ou de prescription [...] ; qu'à une relation politique de nature simple ou complexe, pouvant aller jusqu'à des mesures décisionnelles impliquant la souveraineté d'un État. Cette définition élargie permet à Foucault de dégager les multiples relations et différents paliers de "gouvernement", et ce, avec une attention toute spéciale aux effets qu'ils induisent et produisent au niveau des modes de subjectivation (Malette 2006, p. 59, note 3$)$. 
Cette définition élargie du gouvernement ainsi que le concept de «gouvernementalité» sont proches du sens premier de gouverner et de gouvernement, c'est-à-dire « diriger la conduite de quelque chose ou de quelqu'un ». En effet, gouvernementalité a à voir avec la façon dont le pouvoir s'exerce sur les comportements à travers les discours et les pratiques, autrement dit à travers son action sur la « conduite des conduites ». Si Gautier rappelle à raison que, pour Foucault, l'action sur les conduites, la «conduite des conduites ", s'exerce sur des "sujet-individus libres» (Gautier 2007, p. 23), il importe toutefois de préciser que l'exercice du pouvoir consiste à diriger les conduites, et que gouverner c'est structurer le champ d'action éventuel des autres (Foucault 2001a), donc réduire les libertés.

9 L'une des modalités du pouvoir serait sa capacité à constituer et à réorganiser les représentations des individus, à « normaliser » les écarts :

À essayer de rabattre les normalités les plus défavorables, les plus déviantes par rapport à la courbe normale, de les rabattre sur cette courbe normale, générale. (Foucault 2004, p. 64)

La normalisation "statistique » est donc une forme de remise à la norme et une forme de socialisation des représentations qui réintègre « les normalités les plus déviantes » dans l'ordre social.

11 La notion foucaldienne de gouvernement nous apprend en outre que les formes de gouvernement, loin d'être concentrées au sein du pouvoir exécutif, se subdivisent en " paliers de gouvernement ». Ainsi, il existe différents niveaux de gouvernementalité :

Par «gouvernementalité », j'entends l'ensemble constitué par les institutions, les procédures, analyses et réflexions, les calculs et les tactiques qui permettent d'exercer cette forme bien spécifique, quoique très complexe de pouvoir qui a pour cible principale la population, pour forme majeure de savoir l'économie politique, pour instrument essentiel les dispositifs de sécurité. Deuxièmement, par "gouvernementalité », j'entends la tendance, la ligne de force qui, dans tout l'Occident, n'a pas cessé de conduire, et depuis fort longtemps, vers la prééminence de ce type de "gouvernement" sur tous les autres : souveraineté, discipline, et qui a amené, d'une part, le développement de toute une série d'appareils spécifiques de gouvernement, et, d'autre part, le développement de toute une série de savoirs. (Foucault 2004, p. 111-112).

12 La gouvernementalité est donc une «ligne de force ", laquelle a développé "toute une série d'appareils spécifiques de gouvernement ». Il nous semble que les médias et les discours médiatiques - en tant que discours institutionnels légitimes - sont l'une de ces modalités. Ils contribuent largement à la normalisation des représentations et des savoirs car ils émettent des formes de savoirs qui induisent des normes (des représentations normées), c'est-à-dire des injonctions-prescriptions aux conduites, ils participent ainsi à cette normalisation des conduites ${ }^{4}$.

\subsection{Le rôle de la communication médiatique dans la gouvernementalité}

13 Nous définissons les médias comme des dispositifs technologiques ${ }^{5}$ (presse papier/en ligne, sites Internet, radios, télévisions, blogs, forums, médias sociaux, etc.) permettant une communication de masse et la circulation d'informations publiques d'une instance énonciative réduite vers un plus grand nombre (Guilbert 2015b). Certains de ces dispositifs technologiques font partie de cette «série d'appareils spécifiques de 
gouvernement " qui développent «toute une série de savoirs " dont ils s'adjugent le monopole et/ou l'exclusivité. Sur ce point d'ailleurs, « l'imbrication forte, de plus en plus forte, entre les personnes qui exercent le pouvoir politique, celles qui exercent le pouvoir médiatique et celles qui exercent le pouvoir financier» (Gobin 2010, p.171) et les restructurations monopolistiques continuelles dans la presse sont deux processus liés (Rimbert 2011; Bénilde 2011) qui réduisent le pluralisme de l'information (Cagé 2015) tout en renforçant le pouvoir normatif des médias dominants (Duval 2000). Ce double constat nous conduit à utiliser le terme de " discours politico-médiatique ", lequel signifie qu'il est aujourd'hui de plus en plus difficile de distinguer, dans les discours médiatiques, les sources politiques de leurs commentaires journalistiques (Ringoot 2014; Guilbert 2015a et b).

La sphère politico-médiatique se présente comme l'une des principales détentrices de la « forme majeure du savoir » qu'est « l'économie politique ». Elle connaîtrait les réformes « nécessaires » et saurait ce qui est « bon pour le pays », il n'y aurait plus alors qu’à les lui « expliquer » en faisant preuve de " pédagogie ». Ce savoir exclusif postulé par le discours politico-médiatique dominant correspond à ce que nous avons appelé le « métalangage de la communication » (Guilbert 2011, p. 127) qui est à la fois une forme de refus du débat, donc de négation de la démocratie, et une certaine forme de gouvernementalité.

La forme de gouvernementalité actuelle a donc à voir, semble-t-il, avec ce qu'Alice KriegPlanque appelle la communication :

[...] un ensemble de savoir-faire (plus ou moins professionnels, plus ou moins intuitifs, plus ou moins standardisés...) relatifs à l'anticipation des pratiques de reprise, de transformation et de reformulation des énoncés et de leurs contenus. Cette définition signifie que la communication concerne bien entendu la capacité d'une organisation à faire en sorte que son discours soit répété (comme l'illustre la production de slogans, qui sont de façon très caractéristique des énoncés conçus pour être reproduits). Mais elle signifie aussi que la communication concerne également la capacité d'une organisation à faire en sorte que son discours soit repris en d'autres circonstances, repris par d'autres types de locuteur, repris en d'autres termes, sous d'autres genres et dans d'autres registres: la compétence à communiquer concerne ici la question de la réappropriation des discours (2013, p. 29).

Nous estimons ainsi qu'une communication politico-médiatique réussie possède potentiellement un pouvoir non négligeable sur la constitution et le réaménagement des représentations économiques des citoyens ${ }^{6}$, autrement dit qu'une communication politico-médiatique réussie induit une véritable forme d'hégémonie au sens gramscien du terme ou une « diffusion hégémonique » (Gobin 2010, p. 172).

\subsection{Analyse discursive de crise dans les médias}

Le mot crise est étymologiquement un moment chargé d'une temporalité courte, pourtant les médias utilisent cette temporalité de façon très lâche : le mot crise sert aussi bien à décrire un état quasi permanent depuis plus de 40 ans comme «la crise du chômage ", qu'à nommer un événement soudain et borné dans le temps comme « la crise asiatique de 1999 » ou encore un événement dont on ne sait pas en 2012 s'il va s'installer dans la durée comme «la crise de l'euro » (Guilbert 2017). On est alors proche de la définition que Foucault donne de la notion de crise :

Ces phénomènes d'emballement qui se produisent d'une manière régulière et qui s'annulent aussi de manière régulière, c'est en somme ce qu'on appelle [...] la crise. 
La crise, c'est ce phénomène d'emballement circulaire qui ne peut s'enrayer que par un mécanisme supérieur, naturel et supérieur qui va le freiner, ou par une intervention artificielle. (Foucault 2004, p. 63)

\& Moirand 1995), nous nous intéressons donc à la façon dont ils présentent la crise, aux informations qu'ils fournissent aux citoyens, aux représentations qu'ils leur donnent à intérioriser, c'est-à-dire à la pédagogie de l'économie dans les médias, afin d'en comprendre le but pragmatique qui, selon nous, renvoie à la question foucaldienne: «Comment est-ce qu'on normalise ?» (Foucault 2004, p. 59). Nous réduisons cependant la normalisation à la diffusion des savoirs normatifs de l'économie et reformulons la question ainsi : comment, par quels processus discursifs et sémiologiques, les médias jouent-ils sur nos représentations économiques, donc sur nos conduites? 


\section{Analyse discursive du gouvernement de la crise dans les médias}

\subsection{La crise de 2008 « à chaud » dans la presse française} au lexique des catastrophes naturelles. La métaphore la plus productive est sans nul doute la tempête: "Tempête financière: la contagion frappe à l'Est", "Natixis recapitalise dans la tempête"; "Le monde change, nous sommes au milieu de la tempête » (liberation.fr, 16, 17 et 19/09), "Tim Geithner. Au cœur de la tempête à New York », «La crise des subprimes se transforme en tempête boursière » (lemonde.fr, 17 et 19/09).

Nous illustrerons dans ce paragraphe ${ }^{7}$ le constat selon lequel la majorité de la presse française généraliste ${ }^{8}$ présente au public lors d'une situation de crise deux réponses principales qui sont également deux injonctions aux comportements: la première consiste à gérer la crise en en réduisant les effets, la seconde propose de subir la crise en s'y adaptant, ce qui revient à la considérer comme une catastrophe naturelle inéluctable. Or, non seulement ces deux solutions ne s'excluent pas mutuellement, mais elles nous semblent relever toutes deux d'une même « représentation discursive » de l'événement, c'est-à-dire d'une même forme de présentation et d'une même explication implicite de la crise : un cadrage naturel. De plus, lorsque les médias envisagent la seconde solution comme unique réponse possible, ils signifient implicitement aux citoyens que, face aux crises définies comme imprévisibles et inévitables, l'action politique a peu de pouvoir.

représentatifs 2008 : lemonde.fr et liberation.fr. La temporalité est essentielle ici : la mise en faillite de la banque d'affaire Lehmann Brothers a eu lieu le 15 septembre et crée, selon les médias, le «crash » financier'. Il s'agit donc de montrer comment le moment discursif appelé « crise des subprimes » a été présenté « en temps réel » dans ces deux médias légitimes (Duval 2000). L'analyse de ces énoncés attestés vise surtout à relever les régularités de traitement de l'information entre ces deux journaux et à les comparer aux explications données quelques semaines plus tard par le site lafinancepourtous.fr. Nous intéressons donc, dans les deux corpus, aux procédés discursifs relatifs à la prise en charge énonciative et aux indications données sur les différentes responsabilités, et ce via divers procédés comme la voix active/passive, les verbes pronominaux, le mode agentif, mais aussi les présuppositions, les métaphores, etc.

\section{Les titres}

D'autres formes ${ }^{10}$, moins explicites, font appel également à la tempête en réactivant le topique de la fable de La Fontaine «Le chêne et le roseau »: " Pour la presse, la finance tremble mais ne cédera pas » (liberation.fr, 16/09), «Confrontées au plus grand choc financier depuis la crise de 1929, les places boursières mondiales flanchent, mais ne craquent pas ", «La faillite de Lehman Brothers ébranle le système financier mondial» (lemonde.fr, 16 et 17/09). 
Plus généralement, la crise est présentée comme un cadre de perception naturel, celui d'une catastrophe naturelle. Ce cadre de perception est également produit par le lexique de la maladie ${ }^{11}$, de la violence aveugle ou de l'intervention d'urgence :

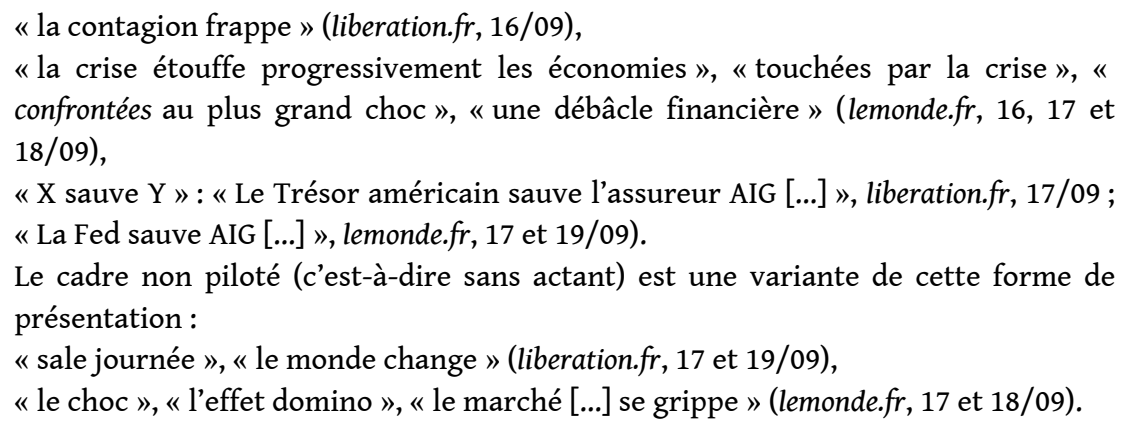

La crise est souvent l'agent de la phrase, c'est elle qui agit et non les agents humains ${ }^{12}$. Nous avons d'ailleurs relevé dans les articles, au-delà des métaphores, le mode d'action proposé et/ou attribué aux agents économiques et politiques: «Vous travaillez dans la finance, comment réagit-on à la crise dans votre entreprise? », « Nos capacités de réaction à une récession sont clairement établies », « M. Sarkozy veut afficher son volontarisme pour compenser son impuissance face à la crise financière » (lemonde.fr, 18 et 19/09).

Enfin, un troisième trait de ces titres concerne le lexique des affects, un lexique inhabituel dans le champ de l'économie politique qui se présente comme un domaine particulièrement rationnel: "Les Bourses mondiales s'affolent", "Wall Street s'inquiète » (lemonde.fr, 16 et 17/09).

L'ensemble des titres constitue un événement sur lequel les agents n'ont aucune prise, sont à la merci d'une catastrophe pour laquelle aucun responsable n'est désigné, aucune cause n'est avancée. La seule réponse à la crise, le seul type de gouvernement de la crise proposé, est d'espérer en être épargné ou d'en atténuer les effets.

\section{Dans les articles}

31 Voici quelques exemples prélevés le 17 septembre 2008, ils illustrent et sont représentatifs de la mise en mots de l'événement dans le corps des articles :

Exemple 1 : lemonde.fr, Claire Gatinois, «Confrontées au plus grand choc financier depuis la crise de 1929, les places boursières mondiales flanchent, mais ne craquent pas ", premières lignes :

LE CHOC provoqué, lundi 15 septembre, par l'annonce de la mise en faillite de la banque d'affaires Lehman Brothers, le rachat précipité de Merrill Lynch, fait vaciller les places financières du monde entier.

Exemple 2 : liberation.fr (avec afp), « Sale journée sur les places boursières ", chapeau :

Le CAC 40 a plongé à son plus bas niveau depuis mai 2005. Les principales places boursières mondiales craignent que la crise financière ne fasse de nouvelles victimes.

Exemple 3 : lemonde.fr, dossier spécial, «Crise financière : les questions clés » :

Quand et comment la spirale d'effondrements bancaires et de séismes boursiers finira-t-elle?

35 Exemple 4 : lemonde.fr, « La crise en questions », chapeau :

Un an après s'être levée aux États-Unis, la tempête des subprimes ravage le système bancaire mondial et menace l'économie réelle 
Les métaphores et analogies dans ces quatre exemples sont fortement isotopiques - c'està-dire d'une grande cohérence ou comportant une forme de dénominateur sémiologique commun (Courtés 1976). Comme pour les titres, elles ressortissent d'un cadre naturel : " choc», «vaciller», «a plongé», «craignent», « victimes ", « spirale», " effondrements ", " ravage ", " séismes », " s'être levée », " tempête », " menace ». Les articles (3 et 4) qui se présentent pourtant par leur titre comme les plus explicatifs et qui, de ce fait, endossent un rôle didactique, sont les plus chargés d'affects et de cadrages naturels. Si l'on trouve des éléments économiques pilotés en 1 («mise en faillite», « rachat précipité »), ce sont des réactions chargées d'affects.

Certes, la plupart de ces métaphores sont éculées et figées ou proches du figement, mais elles n'en perdent pas pour autant leur aspect argumentatif comme l'explique le linguiste Frédéric François :

[...] même lorsque les métaphores sont figées ou proches du figement, la reprise d'un même domaine source dessine une allure de monde et produit un effet spécifique [...] on n'est pas ici du côté de la pure transmission d'informations [...] les métaphores évitent de porter un jugement de valeur explicite. Il s'agit plutôt de présenter les faits « en tant que » (François 2003, p. 11).

Ainsi, les métaphores utilisées ne sont pas seulement explicatives, les énoncés dans lesquels elles apparaissent ont une «visée argumentative » (Amossy 2000), c'est-à-dire une volonté consciente de proposer une certaine vision du monde qui a pour effet possible de réaménager nos représentations. Nous considérons ces métaphores explicatives comme des «analogies argumentatives» (Guilbert 2015c) d'autant plus efficaces qu'étant en partie intégrées à la langue, elles n'en sont que plus facilement mobilisables en discours et paraissent évidentes. Le savoir économique, très réduit, dispensé sur la crise dans ces articles crée une " allure de monde », un monde en crise sur lequel nul ne peut avoir de prise et vis-à-vis duquel il n'y a pas à rechercher des causes mais des moyens de protection.

Il est vrai toutefois que nous avons affaire ici à des discours « à chaud ", les journalistes n'ayant pas le temps du recul sur ce qui se produit alors. Qu'en est-il d'un média qui, contrairement aux journaux étudiés précédemment, a bénéficié du temps nécessaire pour analyser la « crise de 2008 » et qui, de plus, vise à l'expliquer aux citoyens ?

\subsection{La « crise de 2008 » expliquée par lafinancepourtous.fr}

Nous avons choisi d'analyser cette autre forme de média qu'est un site Internet car, à la différence d'un quotidien, il n'a pas à réagir « à chaud ». Les éléments que nous analysons ici sont présentés au public depuis octobre 2008, avec une révision en février 2013. La temporalité est donc bien différente de celle d'un quotidien, et surtout, elle permet un accès à l'information autrement plus important pour les personnes qui alimentent le site.

Par ailleurs, le site se présente comme « Le site pédagogique sur l'argent et la finance ${ }^{13}$, il vise l'éducation financière des citoyens ${ }^{14}$ et est édité par l'Institut pour l'éducation financière du public (IEFP). Jeanne Lazarus, sociologue spécialisée dans l'éducation financière, présente l'IEFP et son site ainsi :

En France, un acteur est officiellement en charge de l'éducation financière: l'Institut pour l'éducation financière du public (IEFP), dépendant de l'Autorité des marchés financiers (AMF), dont la mission est d'améliorer le niveau général de compétences financières de la population. Il est lié aux instances économiques les 
plus légitimes: AMF, Banque de France, ministère de l'Économie et des Finances, ministère de l'Éducation nationale. Pourtant, l'IEFP, connu principalement par son site internet «la finance pour tous", est marginal dans l'espace social de l'éducation financière, car il travaille avant tout avec l'Éducation nationale [...].

(Lazarus 2013, p. 77-78)

Si ce site est certes marginal dans «l'espace social de l'éducation financière » - qui comprend les associations caritatives, les services sociaux et les associations bancaires -, il apparaît pourtant, lors d'une recherche internet sur la crise de 2008, parmi les premiers sites proposés par le moteur de recherche. De plus, comme l'indiquent J. Lazarus et la rubrique "qui sommes-nous?", le site est une source de savoir légitimée par quatre institutions dont deux ministères essentiels ${ }^{15}$. Ainsi, l'agrément de l'Éducation nationale lui confère une forte caution d'objectivité et de didacticité. L'IEFP et son site nous semblent donc faire partie des " appareils spécifiques de gouvernement » par lesquels le savoir économique est diffusé.

Comme tout site, il comporte des liens hypertextuels qui renvoient à des documents internes au site lui-même - des schémas comme «La crise en images » et des textes explicatifs comme "Les cycles financiers" que nous analyserons-, des documents externes mais repris dans le site - un schéma proposé par Le Monde, un film documentaire «Tempête sur la zone euro" (sic) d'Alternatives économiques - et des documents dans d'autres médias. Nous étudions l'identité, la nomenclature du site et le titrage des documents, puis le dossier portant sur la «crise de 2008 ». La nature multimodale des documents proposés par le site nécessite une analyse à la fois discursive et sémiologique.

\section{Étude de l'identité éditoriale du site}

L'identité éditoriale d'un média ne s'inscrit pas uniquement dans les mots utilisés mais aussi et surtout « dans la matérialité du support et de l'écriture » (Souchier 1998, p. 144), c'est-à-dire dans le paratexte : le nom, l'emblème, les couleurs, l'iconographie et, bien sûr ici, les objectifs affichés par le site. Le nom du site La finance pour tous suppose un intérêt frustré des internautes pour ce sujet en jouant sur l'activation d'une représentation posée comme partagée : la finance est un domaine incompréhensible. Ce nom fonctionne alors comme un slogan qui sous-entend une nouveauté, un changement par rapport à un état précédent où la finance n'aurait été comprise que par "quelques-uns». La forme syntaxique du nom du site renvoie à une forme actuelle très prolifique " $\mathrm{X}$ pour tous » («le mariage pour tous» puis, symétriquement, «la manif pour tous»; les sites: orientation-pour-tous.fr, sportpourtous.org, vavances-pour-tous.org, etc.) comportant l'idée de généralisation et de démocratisation d'un domaine ou d'un droit. En montrant ostensiblement sa volonté de vulgariser un savoir et d'aider à la compréhension ${ }^{16}$, le site affiche clairement une forme de didacticité, une visée de transmission des connaissances (Beacco \& Moirand 1995), une forme que nous nommerons « démocratisation didactique » ${ }^{17}$. L'utilisation du soulignement en gras dans la forme graphique du nom « Lafinancepour tous.com » comme dans le sous-titre «Le site pédagogique sur l'argent et la finance » explicitent le domaine par un procédé censé faciliter la lecture et qui est encore un signe de didacticité.

Le pictogramme que le site s'est choisi pour emblème, en haut à gauche de la page, juste avant le nom du site, est un arbre stylisé, légèrement penché comme un bonsaï, dont les feuilles disproportionnées permettent de reconnaître un ginkgo biloba, également surnommé «l'arbre aux mille écus » à cause de la couleur jaune d'or que prennent ses 
feuilles en automne. Cette image d'un bonsaï rappelant à la fois l'esprit zen et l'or participe de la thématique financière et apaisante du site que l'on retrouve dans les couleurs utilisées : orange citrouille, gamme de verts, bleu émeraude, violet; le tout sur fond blanc.

Dans la rubrique « qui sommes-nous? ", la page « La gouvernance et l'équipe » présente les objectifs de l'association ainsi :

Exemple 5 : la finance pour tous, « qui sommes-nous?» :

L'IEFP a été créé pour aider chacun à acquérir les bases de connaissance nécessaires pour :

- se sentir plus à l'aise avec les questions financières,

- comprendre les enjeux économiques du monde dans lequel nous vivons et

- prendre en toute connaissance de cause les décisions qui nous concernent.

La volonté de démocratisation didactique indiquée clairement semble se doubler d'une considération éthique et citoyenne. Pourtant, cette présentation est chargée d'ambiguïté. En effet, un glissement énonciatif se produit quant à la désignation du destinataire : on passe ainsi de chacun, impersonnel et individualisant, à nous ("nous vivons») dont le référent est flou: internautes pris cette fois comme un tout indifférencié, rédacteurs de l'IEFP, citoyens? Du coup, le but réel de cet organisme tel qu'il est formulé ici apparait également flou. Ainsi, l'énoncé « prendre en toute connaissance de cause les décisions qui nous concernent ", et non " qui le concernent ", diffère sensiblement de l'objectif visant à développer les connaissances de chacun. Ce dernier nous renverrait-il aux financiers de l'IEFP ? Aurait-on affaire à un aveu sous forme de lapsus? Il s'agirait alors d'informer les internautes pour qu'ils acquièrent les connaissances nécessaires ${ }^{18}$ afin de prendre les décisions qui concernent... les financiers. L'objectif serait alors de faire adopter aux internautes la « vision IEFP» de la finance. Ce serait une variante du métalangage de la communication (présent par ailleurs dans "comprendre les enjeux économiques»), métalangage qui vise la conduite les conduites.

50 J. Lazarus (2013) permet de mieux comprendre cette auto-présentation et cette ostension de démocratisation didactique. Ainsi, selon l'auteure, l'OCDE prône, depuis le début de la décennie 2000, le «besoin » d'élever le niveau de compétence financière "des classes moyennes salariées». Le terme "financial literacy» a été forgé dans ce but et signifie littéralement «alphabétisme financier». Il «suppos[e] l'existence d'un illettrisme financier " et a été traduit par " éducation financière » : «traduction un peu incomplète car le terme anglais désigne un niveau de compétence plutôt qu'une action éducative " (Lazarus 2013, p. 76). Les « connaissances de base nécessaires » que vise à prodiguer le site renvoient donc à ce « besoin » d'éduquer les classes moyennes.

51 Toutefois, la France a une double particularité. Tout d'abord, elle est l'un des cinq pays de l'OCDE à n'avoir pas intégré dans l'enseignement secondaire un module sur l'éducation financière (Lazarus 2013, p. 77). Ensuite, l'éducation financière y concerne les populations pauvres et ce pour trois raisons : les retraites y fonctionnent encore par répartition et non par capitalisation, la focale est mise sur le surendettement des populations et, enfin, on a mis en place en parallèle le microcrédit qui concerne les plus défavorisés. De ce fait, l'éducation financière française vise les plus pauvres et, pour la Fédération des banques françaises (FBF) comme pour le site la finance pour tous, elle est basée sur le principe de l'inculture en matière de finance des clients, notamment les " "populations fragiles" " (Lazarus 2013, p. 86), faisant ainsi de ceux-ci les responsables des difficultés rencontrées. Il y a donc lieu de les éduquer (Lazarus 2013, p. 77). Selon Georges Gloukoviesoff cité par 
l'auteure, l'objectif didactique mis en avant par ces institutions comporte une visée pragmatique et argumentative dissimulée :

[...] promouvoir l'éducation financière est une manœuvre destinée à masquer la responsabilité des dispositifs et produits bancaires dans les difficultés des clients (Gloukoviesoff 2010, in Lazarus 2013, p. 78).

G. Gloukoviesoff soutient, "en accord avec les néo-foucaldiens britanniques »(ibid., p. 78), que cette éducation financière est un moyen de rejeter les responsabilités, pensées auparavant comme collectives, sur les individus. Cette responsabilisation individuelle est à mettre en relation avec la particularité française de l'éducation financière qui s'adresse prioritairement aux "populations fragiles". Nous verrons que ces deux éléments se combinent dans la culpabilisation des « ménages modestes » à propos de la crise de 2008. Analyse du parcours: Accueil >> Décryptages >> Dossiers >> Crise financière

En arrivant sur la page d'accueil, nous choisissons le premier onglet (à gauche) « Décryptages $»^{19}$, puis dans la page ouverte, la rubrique « Nos dossiers ». Cette dernière propose le choix suivant :

\section{Exemple 6 : la finance pour tous, signet « Décryptages » :}

- Actions

- Immobilier

- Politique de l'offre

- Tous les dossiers indique un hyperlien) : si les deux premiers correspondent en effet à des domaines ou objets liés à l'argent et la finance, le troisième fait figure d'intrus puisqu'il renvoie à une option de politique économique située.

Cliquer sur "Tous les dossiers» permet d'ouvrir une nouvelle page proposant de nombreux dossiers classés par ordre alphabétique de A à U. Nous choisissons "Crise financière » parmi de nombreux autres termes ${ }^{20}$, ce dossier est ventilé en sept rubriques :

Exemple 7 : la finance pour tous, dossier «Crise financière » :

- Les cycles financiers

- Comment la crise 2008 a-t-elle commencé ?

- Comment la crise s'est-elle propagée ?

- Comprendre la crise en images

- L'affaire Goldman Sachs

- Crise financière : quelle protection pour les particuliers

- Le plan de sauvetage européen

Le lexique utilisé semble neutre, voire évident, mais ne l'est pas. Il comporte des métaphores signifiantes qui ont en commun de présenter, comme les journaux que nous avons étudiés, la crise financière comme un cadre naturel non piloté. Ainsi «cycles financiers " présuppose l'existence de lois naturelles fixant des étapes ordonnées comme le cycle des saisons, de l'eau, de la vie, etc. Les deux rubriques suivantes sous forme de questions interrogent le fonctionnement («comment») et non les causes (pourquoi), notamment humaines, de la crise, elles éludent donc les responsabilités (les "pilotes »). La première question donne à "la crise " le rôle de l'agent principal comme une apparition sui generis; la seconde, avec le verbe pronominal se propager, reprend la même idée et $\mathrm{y}$ ajoute une métaphore généralement utilisée lors de catastrophes naturelles ${ }^{21}$ (inondations, incendies, épidémies), les points communs avec les journaux étudiés sont frappants. Les deux dernières rubriques utilisent des métaphores issues du même champ notionnel : «quelle protection pour les particuliers » et «Le plan de sauvetage européen ». 
Toutes deux évoquent une atteinte grave aux personnes par un événement extérieur dont il faut se prémunir. Dans les deux cas, il ne s'agit pas de remédier aux causes de la « catastrophe » mais d'en amoindrir les effets.

Le titre de la fiche "Comprendre la crise en images ", se donne comme particulièrement pédagogique: "comprendre" est associé à "en images", forme censée être plus accessible aux néophytes que les textes. "L'affaire Goldman Sachs» fait figure d'exception mais cette fiche ne dépeint qu'une "affaire " dans l'affaire (la crise) ${ }^{22}$. À l'instar des réactions «à chaud » des deux journaux étudiés, la première perception du dossier « la crise de 2008 ", proposée à l'internaute à travers la nomination des fiches, est celle d'une crise sans responsables (Goldman Sachs n'est pas présenté comme un acteur majeur), d'événements graves qui surviennent d'eux-mêmes.

61 Nous analysons maintenant deux des documents auxquels renvoient ces rubriques, «Les cycles financiers » et « Comment la crise de 2008 a-t-elle commencé ? ", choisis pour leur didacticité. Notre parti-pris méthodologique, celui de l'AD, ne consiste pas à en considérer le contenu (ce qui est dit) et moins encore sa justesse (cela dépasse nos compétences en économie), mais à analyser comment les choses sont dites.

\section{Document $1:$ « Les cycles financiers »}

Cette rubrique se compose d'une introduction - comportant un encadré « Origine d'une crise : Néoclassiques versus Keynésiens » et d'un schéma reprenant le cycle financier - et des cinq phases explicitées (voir Annexe 1).

L'encadré présente, en gras, les deux options opposées et uniques ${ }^{23}$ pour expliquer une crise : "un choc exogène » pour les Néoclassiques, un "choc endogène " pour les Keynésiens. Dans les deux cas, on utilise le même substantif, le choc pour résumer les positions des deux écoles présentées comme antagonistes. Pourtant, le terme choc est impropre et contradictoire avec la présentation de la seconde option: si la crise est " inhérent[e] au système financier par définition instable ", sa cause n'est pas un choc mais l'instabilité même du système, il s'agit une «cause endogène » et non d'un choc. L'utilisation du même mot permet ainsi d'englober dans un même univers notionnel les deux options pourtant adverses, elle confère au mot choc une place centrale en occultant le mot « cause » et conforte, de plus, la métaphore explicative de la catastrophe.

Le texte cite ensuite un certain nombre d'économistes qui se sont intéressés aux cycles de crises économiques ou financières ${ }^{24}$. Cette autolégitimation de la présentation de la crise en cycles met l'accent sur l'un d'entre eux, Charles Kindleberger, lequel a défini les cinq phases détaillées ensuite dans le document: «l'essor, l'euphorie, le paroxysme et le retournement, le reflux et l'instauration du pessimisme et enfin la déflation de la dette et la restructuration des bilans $»$.

Sous le sous-titre "Les cycles financiers » enfin, un schéma est produit qui démontre à nouveau la volonté didactique du site et reprend les cinq phases du cycle.

Étonnamment, le schéma n'a pas une forme circulaire, ce qu'implique pourtant la notion de cycle, mais d'une forme en " $\mathrm{V}$ » inversé qui symbolise une ascension suivie d'une descente, une courbe donc. Les deux premières phases (l'essor, l'euphorie) appartiennent clairement à la partie ascension: elles sont connotées positivement par l'utilisation de flèches montantes et de pictogrammes verts alors que les trois dernières phases descendantes sont orange et violettes. La phase 3, "Paroxysme, retournement», au 
sommet de la courbe est représentée par un éclair orange. Le schéma est saturé d'une redondance signifiante du connoté : les mots et les couleurs désignant les deux premières phases d'ascension sont également connotés positivement, alors que les mots et les couleurs des trois autres phases sont également connotés négativement.

Voyons le texte qui reprend les cinq phases au regard de sa représentation iconographique. Les deux premières phases se composent de deux alinéas : le premier explique en termes généraux la phase en question, le second rapporte celle-ci au cas de la « crise des subprimes».

La représentation iconographique de la phase 1 « essor » dans le schéma est un rectangle comportant un unique pictogramme représentant une maison stéréotypée, type Monopoly, et un stylo-plume ouvert dont la pointe est dirigée en bas à droite du rectangle, l'ensemble vaut pour « signature pour l'achat d'une maison ». Voici l'alinéa 2 :

Exemple 8 : la finance pour tous, « Les cycles financiers » :

Cette phase aurait débuté au début des années 1990 aux États-Unis où l'on constatait un essor de l'octroi de crédits et une phase d'innovation et de libéralisation financière qui s'est traduite par une complexification des produits dérivés et de titrisation notamment ceux adossés aux crédits accordés aux ménages américains.

70 D'un point de vue énonciatif, les actants sont absent de cette explication et ce par différents procédés : la forme impersonnelle utilisée (« cette phase aurait débuté »; «on constatait»; "s'est traduite par») et l'euphémisation "essor de l'octroi de crédits» (pour crédits distribués en grande quantité).

71 Précisons que, à l'instar de A.J. Greimas (1986, p. 130), nous considérons que l'actant syntaxique (le sujet de la phrase ou le complément d'agent) n'est pas forcément l'actant véritable, à savoir celui qui fait l'action - on le constate aisément avec la forme impersonnelle. Mais il faut ajouter aussitôt que c'est la mise en mots de la crise qui nous importe, c'est-à-dire comment l'explication est présentée au public. Or présenter un actant purement syntaxique en lieu et place du véritable actant est très indicatif sur la visée argumentative du site. Ainsi les mots banque, banquiers, bancaire et même organisme de crédit sont totalement absents, ici et dans tout le document.

On peut par contre remarquer la complexité et/ou l'opacité des termes techniques employés et non explicités (« libéralisation financière », « complexification des produits dérivés ", «titrisation »). Ces termes renvoient à un «implicite pour experts» qui n'est "véritablement déchiffré [...] comme il convient que par des lecteurs qui savent "lire entre les lignes" " (Maingueneau 2013, p. 178). Nous avons relevé également une inflation des nominalisations ${ }^{25}$ ("une phase d'innovation et de libéralisation financière ») qui confère au discours un aspect «langue de bois" et un ethos proche de l'«ethos bureaucratique ", c'est-à-dire un ethos " didactique, constamment assertif et qui n'est pas attribué à une source particulière" (Maingueneau 2013, p. 180-181). Ceci confère au discours l'aspect de l'autorité du savoir et du détachement impersonnel de l'objectivité.

73 Pourtant, le lien sémantique entre l'image «achat de maison » et l'explication textuelle "développement boursier» n'est pas clair, il est uniquement suggéré par "ménages américains » complément de "accordés », lui-même épithète du nom "crédits », luimême complément indirect du participe passé «adossés » en fonction d'épithète du pronom « ceux » renvoyant à " produits dérivés ». Cette grande complexité syntaxique en cascade n'est pas fortuite: doublée de la complexité des termes relevée ci-dessus, elle assure l'opacité du discours, et on peut se demander si elle ne mime pas l'opacité du 
fonctionnement de la finance. Cela a pour effet de mettre en lumière les seuls agents désignés à la fois par l'image et par le texte : les « ménages américains ».

La phase 2 «euphorie » est représentée par un temple stylisé et une large flèche verte venant de l'extérieur du « cycle » et apportant des billets de banque qui signifie « apport/ entrée de capitaux dans la finance ». Voici l'alinéa 2 de cette phase :

Exemple 9 : la finance pour tous, « Les cycles financiers » :

C'est ce que l'on a observé [risques sous-évalués ${ }^{26}$ ] avant le déclenchement de la crise des subprimes. Les crédits consentis aux ménages américains étaient surévalués par rapport à leur capacité de remboursement et donc le risque sousévalué. Autrement dit, trop de crédits ont été accordés et les prix des titres financiers adossés à ces créances ne reflétaient pas la réalité du marché.

On peut remarquer à nouveau l'ethos impersonnel et désincarné du texte qui est doublé ici de formes passives sans rappel de l'agent: «déclenchement de la crise » (par qui ?), « étaient sur-évalués » (par qui ?), « et donc le risque sous-évalué » (par qui ?), «ont été accordés » (par qui ?). En soustrayant les agents bancaires, le texte met en exergue les seuls agents présents, les «ménages américains » et « leur capacité de remboursement ». La répétition et la fonction anaphorique de " accordés » renvoient d'ailleurs à la phase 1. Les ménages sont les seuls à être désignés comme responsables de la crise car ils ont choisi de s'endetter. L'adjectif " consentis ", épithète de "crédits ", a ici une importance décisive : il signifie que l'octroi des crédits répond uniquement à leur demande. Cette explication fait bien sûr fi des démarchages et masque, sous le voile pudique du ton impersonnel et de la voix passive, les intérêts financiers des banques pour ces crédits ( subprimes) dont les taux augmentent rapidement : l'origine des fonds n'est d'ailleurs pas précisée par la flèche.

77 L'explication de la phase 3 n'est pas actualisée par la «crise des subprimes » et ne comprend qu'un seul alinéa :

Exemple 10 : la finance pour tous, « Les cycles financiers » :

Cette phase est un point de retournement, le passage d'une période d'euphorie à une période de décroissance. Les anticipations des agents économiques se renversent. Après une période de hausse régulière, on assiste à une chute brutale du prix des actifs.

Comme les phénomènes naturels résultant de lois universelles, les étapes décrites semblent survenir d'elles-mêmes, elles ne sont pas pilotées, elles ressortissent d'un cadre naturel de perception; ce que confirme l'éclair orange qui représente cette phase dans le schéma.

80 L'image de la phase 4 , « le reflux et l'instauration du pessimisme », est le symétrique de la phase 2. Une flèche violette indique que les billets de banque quittent le temple, mais ici leur destination est indiquée: un petit cochon rose symbolisant l'épargne (de qui ?). L'explication textuelle comprend une spécificité : elle comporte un pictogramme rond marqué d'un «! » signifiant «attention ». Ce symbole signale en gras un point important sur le terme " crise », des points de vue économique et métalinguistique :

Exemple 11 : la finance pour tous, « Les cycles financiers » :

Cette phase correspond ici au début de la crise des subprimes. Les prix du marché immobilier américain baissent brutalement.

La crise est d'abord le point de retournement, le passage d'un moment d'euphorie à un moment de décroissance. Par abus de langage, la crise correspond aussi à la période entière de reflux. 
82

Aès les deux phrases qui ancrent l'explication dans le contexte de 2007, sans actants et sans relations directes avec le schéma, la remarque métalinguistique insiste sur l'aspect temporel (la durée restreinte) du sens du mot crise, elle se présente avec sa forme assertive comme une loi de l'économie. représentée par un panneau orange «à vendre » et un gratte-ciel penché comme la tour de Pise, elle n'est pas symétrique de la phase 1 qui montre une maison individuelle. On peut en déduire que le panneau « à vendre » concerne les particuliers et que le gratte-ciel penché représente les établissements financiers pourtant absents dans la phase 1 (si ce n'est peut-être par le stylo-plume qui connote la classe favorisée). Cette phase est décrite par des nominalisations économiques opaques (" une restructuration des bilans », " un assèchement de la liquidité », " la déflation de la dette ») et des énoncés euphémistiques comme :

Exemple 12 : la finance pour tous, «Les cycles financiers » :

Les investisseurs surendettés doivent se désendetter. [...] La dépense privée se contracte

[...]

Le désendettement se réalise beaucoup plus lentement que la chute des prix des actifs ce qui entraîne de nombreuses faillites. d'une explication simple et pédagogique, vantée par le nom du site. Cette page se compose d'un texte entrecoupé par deux images (voir Annexe 2). Nous analysons ici principalement les images car elles sont révélatrices du choix de présentation de la «crise de 2008 ». Dans les textes, on retrouve des éléments similaires au document précédent (voir infra, exemple 13).

La première image est composée de quatre éléments principaux :

- en haut à gauche : un graphique surmonté d'un soleil dans un ciel bleu montrant les «prix de l'immobilier en hausse constante" par une flèche courbe verte ;

- en haut à droite : une maison individuelle avec un arbre (le même que le logo du site) et un bandeau jaune qui traverse toute la maison sur lequel est écrit « achetée »

- en bas à gauche : une silhouette d'homme stylisée de couleur grise avec une cravate noire derrière une inscription verte (avec reflet inversé) « Prêteurs ";

- en bas à droite : un couple, au premier plan la silhouette d'un homme de couleur bleue au second plan la silhouette d'une femme en robe de couleur rose, précédés tous deux par l'inscription en vert (avec reflet inversé) « Emprunteurs » et la mention en petits caractères noirs « ménage modeste ».

Sur ces quatre images, un certain nombre de flèches vertes indiquent des relations économiques : une flèche du couple vers la maison intitulée « Achat d'un logement » et 
deux flèches, l'une de l'homme gris vers le couple nommée dans un cercle «Credit subprime » (premier mot en orange, second en petites capitales blanches), l'autre dans le sens inverse nommée "Charges financières très allégées au démarrage ». Une explication complémentaire et technique est apportée sous «Credit subprime » en gras et en noir : "Taux d'intérêts moins préférentiels, Taux variables gagés sur une hypothèque, Crédits rechargeables ».

Cette iconographie mérite quelques remarques. Tout d'abord, les images sont gorgées de connotations stéréotypées : la situation générale positive (beau temps et couleurs vives), la représentation des sexes (bleu pour l'homme, rose pour la femme, homme au premier plan et plus grand, femme au second plan et plus petite, le prêteur est un homme), les relations sociales (vêtement gris et cravate noire opposé à vêtement bleu (« col bleu»?), le prêteur légèrement plus grand que l'homme emprunteur; distinction de chaque jambe dans les silhouettes du couple/non distinction et aspect monolithique pour le prêteur). Ces connotations sont redondantes avec les éléments verbaux inclus dans les images. La mention euphémistique «ménage modeste » masque qu'il s'agit «des classes les plus pauvres », les minorités souvent « afro-américaines » (Dollé 2009, p. 78).

Ensuite, cette image prend des libertés vis-à-vis de l'objectivité des faits. Ainsi, l'aspect positif de la période d'essor ne rend pas compte du fait que la « crise des subprimes » a commencé un an avant la crise de 2008. C'est peut-être le reflet d'un sentiment partagé en 2008, mais ceci est présenté comme un fait. De même, la mention "achetée » sur la maison ne correspond pas à la routine sociodiscursive: on indique généralement "vendue » sur une maison et non "achetée »; elle renvoie implicitement à un procès achevé alors qu'il s'agit justement d'emprunts (acheter à crédit n'est pas posséder). Cela constitue donc à la fois une euphémisation de la réalité - comme "Charges financières très allégées au démarrage » et "Taux d'intérêts moins préférentiels » - et un moyen de diriger l'attention vers les "acheteurs" (ménages) et non vers les "vendeurs" (organismes de crédit et banques).

92 La seconde image reprend ces quatre éléments mais bouleversés par la crise :

- le graphique en haut à gauche est inversé : la flèche courbe va vers le bas, passe du vert à l'orange et est surmontée d'un ciel gris d'orage chargé de nuages et d'éclairs ;

- l'unique maison est démultipliée et chacune comporte un bandeau avec la mention «à vendre », l'arbre est vert foncé ;

- le prêteur passe du gris au rouge, il est en train de tomber, désarticulé et propulsé par le cercle « Credit subprime » devenu boule, comme dans un jeu de quilles;

- le couple maintenant de couleur rouge se tient debout, tête baissée.

Les flèches-relations sont devenues rouges également : l'une va du couple aux maisons et indique " perte du logement ", l'autre, beaucoup plus grosse, va du couple au prêteur et précise « les charges de remboursement s'alourdissent». Deux autres mentions ont été ajoutées l'une sous le prêteur stipule «Les établissements spécialisés en crédit se sont, les premiers, retrouvés en difficulté ", l'autre "Taux de défaillance des emprunteurs U.S. : $15 \%$ en 2007 ».

La présentation des faits est à nouveau partiale. Tout d'abord, deux éléments sont contrefactuels: la flèche «les charges de remboursement s'alourdissent» est curieusement dirigée des ménages vers le prêteur; la mention «Les établissements spécialisés en crédit se sont, les premiers, retrouvés en difficulté » (reprise du texte) font fi des difficultés rencontrées par les milliers de ménages américains. En effet, ceux-ci se 
sont retrouvés sans maison. Or pour les Anglo-Saxons, une maison « constitue la forme la plus élémentaire d'exister en propre, et de se situer dans le monde", elle permet d'« acquérir la propriété de soi-même, et par là même, [de] conquérir sa liberté » (Dollé, op. cit., p. 78-79). C'est donc une forme de subjectivation impossible qu'ont subie «les membres des minorités afro-américaines descendant des esclaves, et des LatinoAméricains, enfants des péons des latifundias, semi-serfs soumis à une domination de type féodal », c'est-à-dire l'impossibilité d' « intégrer la fameuse classe moyenne » (ibid.).

Ensuite, l'iconographie et les mots mettent l'accent de façon convergente sur le rôle préjudiciable des ménages qui auraient mis en danger les prêteurs : par la grosse flèche rouge, le sens contrefactuel de la flèche et la chute du prêteur d'une part, par l'incise « les premiers " et la nominalisation "défaillance des emprunteurs " ${ }^{28}$, d'autre part. Cette seconde image montre à nouveau une responsabilité forte des ménages tout en occultant leurs difficultés.

Le chapeau de l'article, qui accompagne le texte et cette iconographie, indique clairement d'ailleurs, comme dans le document précédent, cette responsabilité des ménages :

Exemple 13 : la finance pour tous, « Comment la crise de 2008 a-t-elle commencé ? » :

La crise de 2008 a débuté avec les difficultés rencontrées par les ménages américains à faible revenu pour rembourser les crédits qui leur avaient été consentis pour l'achat de leur logement.

On retrouve l'utilisation des mots ou idées rencontrés dans le document 1 ou dans l'iconographie des documents 1 et 2 : la reprise de «ménages américains » (ex. 8, 9 et 13); " crédits [...] consentis " renvoie à la fois à "crédits consentis» (ex. 9) et «crédits accordés » (ex. 8 et 9); "ménage [...] à faible revenu " rappelle "ménage modeste» (image 1) ; "achat» entre en écho avec le bandeau "acheté » sur la maison. Ces répétitions assurent à nouveau une forte isotopie textuelle et iconographique.

Enfin, les formes syntaxiques, outre qu'elles assurent l'ethos institutionnel et didactique du texte, renforcent la focalisation sur les ménages et l'occultation des responsables. Ainsi l'utilisation de la forme passive incluse dans une longue nominalisation qui commence par «avec» et qui court jusqu'à la fin de la phrase résulte d'une double transformation :

- transformation voix active/voix passive :

- Les ménages américains à faible revenu ont rencontré des difficultés pour rembourser leurs crédits devient: Des difficultés ont été rencontrées par les ménages américains à faible revenu pour rembourser leurs crédits.

- nominalisation de la voix passive (transformation du groupe verbal en groupe nominal) :

- Les difficultés rencontrées par les ménages américains à faible revenu pour rembourser leurs crédits...

Cette double transformation - dont on peut douter raisonnablement qu'elle est fortuite met à nouveau en avant le seul agent humain de la phrase, "ménages américains", introduit par la préposition " par » et permet d'accrocher à « crédits » la subordonnée à la voix passive: "qui leur avaient été consentis ». Le véritable agent de cette seconde action (chronologiquement la première) est à nouveau occulté. Dans la suite de l'article, le prêteur n'est pas plus nommé, les rédacteurs ont utilisé :

- la forme impersonnelle avec nominalisation («L'endettement des ménages américains a pu s'appuyer sur »),

- la voix passive (« Les crédits "subprime” ont été gagés par une hypothèque sur le logement acheté ») 
- ou encore la non prise en charge par un on indéfini (« on prenait en compte la hausse de la valeur du bien, et on autorisait l'emprunteur à se réendetter... »).

Encore une fois, le seul agent humain dans le texte de ce document est « l'emprunteur » ou « les ménages américains », repris par des possessifs (« leurs », « son », « ses », etc.).

D'un point de vue sémiologique, cette responsabilisation des ménages se double de l'attitude, hautement signifiante, essentielle et à nouveau isotopique que l'iconographie donne au prêteur et au couple-emprunteur : la chute du premier marque son absence de responsabilité, voire son statut de victime, alors que la tête baissée des seconds mime non pas la responsabilité mais la culpabilité et la honte. Les ménages modestes américains ne sont donc pas seulement responsabilisés mais culpabilisés, la honte semblant être l'attitude à la fois idoine et prescrite.

3 Conclusionil y aurait encore beaucoup à dire et beaucoup à analyser dans le site, qui propose, par exemple dans la rubrique "La crise en images », des animations, un schéma du Monde ou encore un documentaire, "Tempête sur la zone euro", réalisé par le périodique Alternatives économiques. Dans ce dernier média, pourtant considéré comme plus hétérodoxe qu'orthodoxe en économie, on retrouve, comme dans le reste du site, le lexique des catastrophes (par exemple "tempête » et l'utilisation récurrente du verbe «subir ») mais aussi une dénonciation de la dette et de l'endettement, comme le montre la déclaration suivante de Sandra Moatti, directrice en chef adjointe: des pays qui ont dépensé massivement plus qu'ils ne gagnaient et c'est sur ces conditions de possibilité qu'il faut s'interroger (nous soulignons)

On trouve donc dans les médias ${ }^{29}$ deux représentations principales et complémentaires de la crise 2008, représentations que l'on peut résumer, d'une part, à une catastrophe naturelle et, d'autre part, à la faute et la culpabilisation des ménages et des États pauvres. On pourrait n'y voir que des routines, des schématisations journalistiques ou un panurgisme médiatique, mais en $\mathrm{AD}$, il importe de rapporter les discours analysés et les représentations qu'ils véhiculent à leur "formation discursive", c'est-à-dire à la fois à leur « source ", leur « archive ", leur « centre généalogique » ou encore à l'ensemble de discours qui ont une position idéologique commune ${ }^{30}$.

Ainsi, le cadre naturel de perception (une catastrophe inéluctable) dans lequel la crise est présentée par les médias produit l'occultation des responsabilités par un procédé de désincarnation des responsables, il est la face visible et évidente d'un discours idéologique qui vise à masquer les responsabilités ${ }^{31}$. Il participe de la naturalité discursive propre à l'appareillage de l'idéologie libérale (Keynes 1999 [1926] ; Polanyi 1983 [1944]) et au discours néolibéral (Guilbert 2007, 2011).

De même, l'éducation financière des citoyens préconisée par l'OCDE et la responsabilisation des "ménages modestes" et des pays pauvres qui apparaît plus tardivement dans les discours médiatiques appartiennent à la même formation discursive, à cette " conception libérale de la responsabilité individuelle » (Gautier, op. cit ., p. 33) présente dans le néolibéralisme ou dans l'économisme (Viveret 2001) mais aussi dans l'ordo-libéralisme européen. Quasi absente des journaux qui, on l'a vu, sont au cœur de l'événement mi-septembre 2008, cette responsabilisation des ménages est omniprésente dans le dossier «Crise de 2008 » du site, écrit le 22 octobre 2008 et revu le 7 février 2013. Cette construction de la responsabilité "des ménages à faible revenu " apparaît donc dans un second temps et s'appuie sur la disparition « à chaud » des agents. La responsabilisation/culpabilisation s'est ensuite élargie, dans des « explications » des 
«crises » de l'euro, de la dette et de la Grèce, aux pays et aux États pauvres, comme l'illustre le propos de S. Moatti ci-dessus. Ces deux dénonciations sont d'ailleurs de même nature, leur différence ne tient qu'à une question de degré ou d'échelle : « $\mathrm{X}$ a vécu audessus de ses moyens » est le topique de la dette, une accusation d'irresponsabilité, qu'on a pu entendre dans les discours politico-médiatiques tant à propos des ménages que des peuples grecs, portugais, espagnols, français, etc.

Cependant, les discours néo- et ordo-libéraux ne sont qu'une partie des sources ou des formations discursives dont est issu ce discours, l'ouvrage La haine de la démocratie de Jacques Rancière (2005) permet d'en distinguer une autre. Rancière montre que la démocratie a "identifié sans plus de façon l'égalité démocratique à l'"égal échange" de la prestation marchande » (ibid., p. 26). Ce qu'il nomme «la haine de la démocratie » est la dénonciation actuelle dans le monde politico-médiatique de l'excès de démocratie et de «l'individualisme démocratique ». Selon l'auteur, la dénonciation de l'excès de démocratie rassemble deux thèses: la thèse très ancienne des "possédants (les pauvres veulent toujours plus) $»^{32}$ et la thèse plus récente des «élites raffinées: il y a trop d'individus, trop de gens qui prétendent au privilège de l'individualité ». La synthèse de cette double thèse aboutit, selon Rancière, à la dénonciation selon laquelle nous serions aujourd'hui dans un « individualisme de masse » totalement inconséquent (ibid., p. 35-37).

Cette thèse avait déjà été défendue dans La révolte des masses, ouvrage paru en 1930 et vendu en deux ans à plus 120000 exemplaires en Espagne ${ }^{33}$ :

Ce qui est déplorable est que cette décision prise par les masses d'assumer des activités propres aux minorités, non seulement ne se manifeste et ne peut se manifester que dans l'ordre des plaisirs, mais qu'elle est le signe général des temps. [...] Aujourd'hui nous assistons au triomphe d'une hyper-démocratie dans laquelle la masse agit directement sans loi, imposant ses aspirations et ses goûts au moyen de pressions matérielles. (Ortega y Gasset 2010, p. 89)

L'irresponsabilité et la responsabilisation « des ménages à faible revenu » qui ont "vécu au-dessus de leurs moyens" reconduisent cette double dénonciation issue d'une formation discursive conservatrice. La stigmatisation des ménages modestes américains très présente dans le site lafinancepourtous.fr actualise cette forme de culpabilisation des pauvres: "les "ménages modestes" prétendent maintenant eux aussi, de manière totalement inconséquente, acheter une maison individuelle». Les expressions euphémistiques "à faible revenu ", "ménage modeste » et l'iconographie de la honte coupable ont ici toute leur importance, de même que les participes passés adjectivés "consentis " et "accordés » en position d'épithète et de "crédits » qui marquent implicitement l'inconséquence de ces derniers. Ces expressions construisent des représentations qui blâment le désir consumériste et les dettes des "ménages modestes " et qui les assignent, implicitement, à leur condition sociale, voire à leur «minorité raciale » (Dollé 2009) : leur culpabilisation est une façon de sous-entendre qu'ils ne sont pas restés à leur place, elle leur dénie le droit à l'individualité et la prétention à l'achat d'une maison.

110 Plus largement, ce discours économico-médiatique chargé d'autorité et de légitimité fonctionne comme un métalangage de la communication en surplomb de la plupart des discours des autres agents économiques. Ce discours qui vaut aussi pour les pays du sud de l'Europe dont les peuples et les États auraient " vécu au-dessus de leurs moyens » et ne se seraient pas comportés en «bons débiteurs » (Guilbert 2015a), participe de fait à une forme de gouvernementalité qui les culpabilise également; doublé de «la rhétorique 
naturalisante de la crise », il est « le moyen d'intimidation d'un pouvoir » (Vollaire 2009, p. 66).

111 Le politiste et psychanalyste Yannis Stavrakakis (2013) explique que nous serions passé « du jour au lendemain » d'une société du crédit à une « économie » ou une " société de la dette " ("debt economy », "debt society») en utilisant la culpabilisation de l'endettement comme instrument de pouvoir (Stavrakakis 2013, p. 39). Partant de ce constat et de nos analyses, nous posons que "gouverner la crise» de 2008, via les discours politicomédiatiques sur l'économie politique, consiste à produire «un consensus intellectuel dominant » (Rancière 2005, p. 94) à propos de la « crise » : une occultation substituant la responsabilité des citoyens (pauvres) à celle de la sphère financière. Ainsi l'IEFP, en tant qu' "appareil spécifique de gouvernement" et à travers son site lafinancepourtous.fr, "gouverne la crise » en la présentant comme une catastrophe naturelle et agit sur les conduites en désignant les "mauvais comportements» fauteurs de crises - comme la surconsommation et le surendettement des «ménages modestes». La pédagogie économique est alors, comme le pense Gloukoviesoff, un moyen de rejeter sur les individus les responsabilités pensées auparavant comme collectives. Cette construction de la crise participe alors à la mise en place d'un complexe de culpabilité/honte («guilt/ shame complex ») qui consiste à créer la honte par la culpabilisation et qui est un moyen de préparer, au nom de la raison économique, à la punition («punishment») des coupables (Stavrakakis 2013, p. 38). Autrement dit, la culpabilisation, comme l'urgence induite par le mot crise, préparerait la soumission des ménages aux politiques économiques austéritaires ${ }^{34}$.

Ces deux représentations de la crise de 2008 (phénomène naturel et responsabilisation des ménages) semblent donc fortement interreliées mais peuvent sembler paradoxales: comment en effet un discours qui se veut rationnel peut-il soutenir à la fois la naturalité des crises et la responsabilisation-culpabilisation des ménages vis-à-vis de ces mêmes crises? Le premier élément de réponse est que ce discours n'est ni homogène, ni uniforme et que s'il vise à se montrer rationnel, il n'y a pas de raison de penser qu'il l'est forcément. Peut-être a-t-on affaire à une pensée magique et superstitieuse : les «mauvais comportements » auraient déclenché des forces maléfiques jusque-là endormies. Plus sérieusement, on peut, second élément de réponse, faire l'hypothèse que cette présentation médiatique produit une forme de double contrainte, une injonction paradoxale de gouvernement des conduites qui culpabilise le consumérisme des dominés, ceux qui consomment le moins, après leur avoir vanté les vertus du crédit, et qu'elle résulte d'un processus discursif à deux temps: un cadrage naturel qui déresponsabilise les responsables suivi d'une culpabilisation des victimes.

\section{BIBLIOGRAPHIE}

Althusser L. (1976) [1970], « Idéologie et appareils idéologiques d’État. Notes pour une recherche ", in Positions, Paris, Éditions Sociales, p. 67-125. 
Amossy R. (2000), L'argumentation dans le discours. Discours politique, littérature d'idées, fiction, Paris, Nathan université.

Bénilde M. (2011), « Patrons de presse en campagne », Le Monde diplomatique, septembre.

Beacco J.-C. \& S. Moirand (1995), « Autour des discours de transmission de connaissances », Langages, $\mathrm{n}^{\circ} 117, \mathrm{p} .32-53$.

Cagé J. (2015), Sauver les médias. Capitalisme, financement participatif et démocratie, Paris, Le Seuil, coll. « La république des idées ».

Charaudeau P. (1997), Le discours d'information médiatique. La construction du miroir social, Paris, Nathan-INA.

Courtés J. (1976), Introduction à la sémiotique narrative et discursive, Paris, Hachette.

Dollé J.-P. (2009), « La crise est là », Lignes, n 30, p. 78-88.

Duval J. (2000), « Le journalisme à l'économie », Actes de la recherche en sciences sociales, 131-132, p. 56-75.

Fairclough N. (1995), Critical Discourse Analysis: The Critical Study of Language. Edimburg, Longman Group Limited.

Foucault M. (1971), L'ordre du discours, Paris, Gallimard.

Foucault M. (2001a), « Le sujet et le pouvoir », in Dits et Écrits, vol. 2 : 1976-1988, Paris, Gallimard, coll. « Quarto».

Foucault M. (2001b) [1966], Les mots et les choses, Paris, Gallimard, coll. « Tel ».

Foucault M. (2004) [1977-1978], Sécurité, territoire, population, Paris, Le Seuil.

François F. (2003), « Préface », in R. Kerzazi-Lasri (dir.), La Métaphore dans le commentaire politique, Paris, L'Harmattan.

Gautier C. (1997), «À propos du "gouvernement des conduites" chez Foucault : quelques pistes de lecture », in J. Chevallier (dir.), La gouvernabilité, Paris, PUF, coll. « Curapp », p. 19-33.

Gloukoviesoff G. (2010), L'Exclusion bancaire, Paris, PUF.

Gobin C. (2010), « Des principales caractéristiques du discours politique contemporain... », Semen, $\mathrm{n}^{\circ} 30$, p. 169-186.

Greimas A.J. (1986) [1966], Sémantique structurale. Recherche de méthode, Paris, PUF.

Guilbert T. (2007), Le discours idéologique ou la force de l'évidence, Paris, L'Harmattan, coll.

«Logiques sociales».

Guilbert T. (2011), L'« évidence » du discours néolibéral. Analyse dans la presse écrite, Bellecombe-enBauges, Éditions du Croquant, coll. « Savoir/agir».

Guilbert T. (2015a), « “Crise de l'euro”, analyse d'une formule et de ses effets », Économie et institutions [En ligne], 22 | 2015, mis en ligne le 01 juin 2015, consulté le 19 janvier 2018. URL : http://journals.openedition.org/ei/994 ; DOI : 10.4000/ei.994

Guilbert T. (2015b), « Leonarda, le Président et les médias. Communication politico-médiatique et représentations identitaires à propos des Roms ", in F. Hailon, A. Richard \& N. Guellil (dir.), Le Discours politique identitaire dans les médias, Paris, L'Harmattan, coll. « Questions contemporaines », p. 77-108. 
Guilbert T. (2015c), «L'analogie argumentative et la constitution des "évidences" : analyse de la presse en ligne dans la "tempête des subprimes" ", à disposition sur academia.edu, lien : https:// www.academia.edu/people/search?utf $8=\checkmark \& q=$ analogie+argumentative

Guilbert T. (2017), « Analyse discursive et sémiologique du mythe "crise grecque”. La naturalisation par le récit dans Les Échos en 2010 », in C. Constantopoulou \& B. Péquignot (dir.), Les récits de la crise économique : mythes et réalités de la société contemporaine, Paris, L'Harmattan, coll. « Logiques sociales », p. 131-155.

Guilbert T. \& Lebaron F. (2017), « L'économie des mots et les mots de l'économie, analyse sociodiscursive des discours des dirigeants de la Banque centrale européenne ", Langage \& Société, 160-161, « Langues, langages et discours en sociétés. La revue a 40 ans », p. 217-235.

Keynes J.M. (1999) [1926], The End of laisser-faire, Marseille, éditions Agone, coll. « Contre-feux », p. 3-36.

Krieg-Planque A. (2013), Analyser les discours institutionnels, Paris, Armand Colin.

Laborit H. (1974), La nouvelle grille, Paris, Folio-Essai.

Lazarus J. (2013), « De l'aide à la responsabilisation. L'espace social de l'éducation financière en France ", Genèses, vol. 4, n 93, p. 76-97.

Lignes 2009/3 (30), « De la crise comme méthode de gouvernement », http://www.cairn.info/ revue-lignes-2009-3.htm

Maingueneau D. (1995), « Présentation », Langages, 117, p. 5-11.

Maingueneau D. (2013), « Post-scriptum : le rapport de la Banque mondiale. Quelques réflexions d'un analyste du discours », in M. Temmar, J. Angermüller \& F. Lebaron (dir.), Les discours sur l'économie, Paris, PUF, coll. « Curapp », p. 175-195.

Malette S. (2006), La « gouvernementalité » chez Michel Foucault, Faculté des études supérieures, Université Laval, Québec.

Moirand S. (2007), Les discours de la presse quotidienne : observer, analyser, comprendre, Paris, PUF.

Ortega y Gasset J. (2010) [1930], La révolte des masses, Paris, Les Belles Lettres.

Pêcheux M. (1969), Analyse automatique du discours, in D. Maldidier (1990), L'inquiétude du discours. Textes de Michel Pêcheux, Paris, Éditions des Cendres, coll. « Archives du commentaire », p. 98-132. Polanyi K. (1983) [1944], La Grande Transformation, Paris, Gallimard.

Rancière J. (2005), La haine de la démocratie, Paris, La fabrique éditions.

Rey A. (1998) [1992], Dictionnaire historique de la langue française, Le Robert en trois volumes.

Rimbert P. (2011), « Médias et politiques, trêve de complaisance ? », Le Monde diplomatique novembre.

Ringoot R. (2014), Analyser le discours de presse, Paris, Armand Colin.

Stavrakakis Y. (2013), « Debt society. Greece and the future of post-democracy », Radical Philosophy, 181, p. 33-38.

Souchier E. (1998), « L'image du texte. Pour une théorie de l'énonciation éditoriale », Cahiers de médiologie, $\mathrm{n}^{\circ}$ 6, p. 137-145.

Viveret, P. (2001), « Reconsidérer la richesse, Le rapport d'étape », Transversales Sciences - Culture, $\mathrm{n}^{\circ}$ 70, p. 4-36. 
Vollaire C. (2009), « Injonctions léthales », Lignes, $n^{\circ} 30$, « De la crise comme méthode de gouvernement », p. 58-66.

\section{NOTES}

1. Gouverner suppose une certaine durée et une maitrise humaine, tandis que crise renvoie à un événement ponctuel et sui generis.

2. Bien que les trois termes ne soient pas synonymes, nous alternerons entre didacticité, pédagogie et éducation, selon les sources. Le premier terme est employé en sciences du langage, le deuxième par le site étudié et le troisième par J. Lazarus. Il est possible que l'on trouve ces trois pans de la transmission de connaissances sur le site, mais ce n'est pas l'objet ici de les distinguer formellement.

3. C'est le deuxième objectif poursuivi par Foucault. Le premier et le troisième sont : «l'étude du développement du gouvernement en Occident comme type de pouvoir distinct de la souveraineté et de la discipline » et « l'analyse du processus par lequel l'État de justice du Moyen-Âge, devenu "État de police" aux $\mathrm{XVI}^{\mathrm{e}}$ et $\mathrm{XVII}^{\mathrm{e}}$ siècles, s'est progressivement "gouvernementalisé" " (ibid., p. 58).

4. Les médias ne sont bien sûr pas les seuls à le faire. Nous avons proposé ailleurs cette normalisation comme l'un des traits principaux du discours économique (Guilbert \& Lebaron 2017).

5. Nous les distinguons des objets technologiques (ordinateurs, tablettes, smartphones, etc.).

6. Cette formulation laisse entendre une position politico-médiatique dominante : nous avons pu la mettre en évidence lors des mouvements sociaux de 1995 et 2003 (Guilbert 2007, 2011). Le même constat peut être fait lors du référendum de 2005 et de la « réforme » des retraites de 2010. 7. Nous nous appuyons implicitement sur certains de nos travaux récents (Guilbert 2015a, 2015c, 2017) sans les citer systématiquement.

8. Voir Guilbert $(2015 \mathrm{c})$. Certains font exception comme Manière de voir, publication du Monde diplomatique, qui titre « Le casino et le cantonnier » à propos de la « crise de 2008 ».

9. «Le 15 septembre 2008, Lehman Brothers se déclare en faillite. Avec 639 milliards de dollars d'avoirs, une dette de près de 619 milliards de dollars et 25000 employés dans le monde, Lehman était la quatrième banque d'investissement américaine. »https://www.cafedelabourse.com/ dossiers/article/etude-de-cas-la-chute-de-lehman-brothers (consulté le 11/10/2017).

10. C'est nous qui soulignons dans ces titres.

11. C. Vollaire (2009, p. 58) rappelle que le mot crise est né dans « le domaine médical » et qu'on le trouve la toute première fois dans le livre des Épidémies.

12. En voici un autre exemple : «prochaines victimes de la crise?» (lepoint.fr, 17/09/2008).

13. Depuis la date d'analyse de ce site (décembre 2015), certains documents ont changé. Ainsi le document "Comment la crise a-t-elle commencé ?", que nous analysons ci-après, a été revu en février 2016. Nous donnons quelques indications sur le nouveau document ci-dessous mais conservons l'analyse du document présenté au minimum de février 2013 à février 2016.

14. D'autres sites existent bien sûr, voir par exemple cafedelabourse.com, acheteror.org ou encore Mataf.net.

15. Et : « L'IEFP est une association d'intérêt général indépendante, créée en 2006. Ses principaux soutiens sont l'Autorité des marchés financiers, la Fédération bancaire française, la Banque de France et le ministère de l'Éducation nationale (l'IEFP est agréé par le ministère). »

16. On retrouve la même idée dans ce lien proposé par Mataf.net : https://www.mataf.net/fr/ bourse/edu/apprendre-la-bourse/la-faillite-de-lehman-brothers-expliquee-tout-simplement (consulté le 11/10/2017). 
17. Il y a également une visée de "démocratisation financière » dans le projet d' "éducation financière » de l'OCDE (voir infra) : augmenter le nombre de citoyens-actionnaires.

18. Adjectif récurrent du discours néolibéral (Guilbert 2007).

19. Ce terme est encore une ostension de didacticité, c'est aussi un nom de rubrique commun avec la presse.

20. Nous avons cherché des items en relation avec "gouverner » et n'avons trouvé ni État, ni gouverner, ni gouvernance, ni gouvernement. D'ailleurs, rien n'est proposé à la lettre «g ", on passe directement de «Fonds monétaire international (FMI)» à «Hautes rémunérations en France ".

21. On retrouve ce cadrage naturel dans le chapeau de ce document : "Le déclencheur de la crise se situe donc dans un sous-compartiment du marché immobilier américain. Les établissements spécialisés dans le crédit "subprime" ont été directement et logiquement touchés. Mais c'est la mutation opérée depuis une quinzaine d'années avec ce que l'on appelle la titrisation qui permet de comprendre pourquoi et comment la contagion s'est opérée. » (nous soulignons)

22. Ce document utilise des termes si techniques qu'il est incompréhensible pour un néophyte.

23. L'analyse des crises du capitalisme a pourtant été étudiée par une autre option, celle de Marx (par ex : Vollaire 2009, p. 61-62).

24. «Le concept de crise est lié à l'observation de la cyclicité du temps naturel [...]» (Vollaire 2009, p. 58).

25. Une nominalisation est la transformation d'une phrase verbale en phrase nominale. Exemple : l'économie est en crise devient la crise de l'économie. L'intérêt persuasif de cette forme est de favoriser les présuppositions (Guilbert 2011, p. 47-49).

26. Le site ne précise pas par qui.

27. Le document analysé est celui qui a été présent de février 2013 à février 2016 (voir annexe). Depuis, les deux images analysées ont été remplacées par une seule mais la mention « ménages à faibles revenus " y demeure, le texte a été peu modifié et conserve sensiblement les mêmes traits.

28. Dans le corps du texte, l'euphémisation et l'effet sui generis sont présents également : «L'afflux des défaillances des emprunteurs [...]. »

29. Ceux que nous avons étudiés bien sûr mais ils nous semblent très représentatifs des représentations dominantes.

30. Des discours qui résultent d'un même "processus de production dominant régissant le discours soumis à l'analyse. » (Pêcheux 1969, p. 128). Cette notion renvoie donc pour nous aussi bien à la notion forgée par Foucault qu'à sa reprise et à son interprétation par Pêcheux.

31. Voir sur ce sujet l'article de C. Vollaire «Injonction léthales » dans le $n^{\circ} 30$ de Lignes.

32. L'injure: "Salauds de pauvres!", lancée par le personnage que joue Jean Gabin dans La traversée de Paris, se situe dans le cadre de cette très vieille thèse des « possédants ".

33. Il a en outre été traduit dans toutes les langues européennes et a été vendu à plus de 300000 exemplaires en Allemagne. «De nos jours, [...] la pensée et l'œuvre d'Ortega demeurent vivantes » en Espagne et en Amérique du Sud (Argentine, Pérou, Colombie, Mexique) à travers la Fondation Ortega y Gasset et des Instituts universitaires ». J.L. Goyena, Préface à l'édition de 2010, p. 43-44.

34. Notre analyse de l'urgence contenue dans les formules politico-médiatiques «crise de l'euro » et « crise de la dette » en 2012-2013 (Guilbert 2015a) parvenait à une conclusion similaire, ce qui montre l'aspect protéiforme de cette formation discursive. Sur l'urgence et la soumission, voir aussi C. Vollaire : «L'usage du concept de crise est ici un processus de naturalisation du politique, qui permet à la fois la neutralisation de toute 
responsabilité originelle, et l'émergence d'une sorte de fatalisme effaré qui pousse à la soumission. » (2009, p. 60).

\section{RÉSUMÉS}

Cet article analyse de façon discursive et sémiologique comment les discours médiatiques ont participé au " gouvernement des conduites » pendant et après de la « crise de 2008 ». La notion foucaldienne de gouvernementalité y est revisitée dans le cadre de l'analyse de discours en prenant en compte le rôle et l'importance de la communication politico-médiatique actuelle. L'analyse porte sur le premier temps du « gouvernement de la crise » dans deux quotidiens de la presse française du 16 au 19 septembre 2008 et montre que la crise y est présentée comme une catastrophe naturelle, ce qui occulte les responsables de la crise. Puis, pour illustrer le second temps, l'analyse étudie la présentation légitime de la crise par le site pédagogique de l'Institut pour l'Éducation Financière du Public (IEFP), lafinancepourtous.fr. Elle met en lumière une focalisation sur les "ménages modestes" qui les désigne à la fois comme responsables et coupables.

This article analyzes in a discursive and semiological way how some media discourses participated in the "government of conduct" during and after the "crisis of 2008". The Foucauldian notion of governmentality is revisited in the discourse analysis, taking into account the role and importance of current politico-media communication. The analysis focuses on the first stage of the "government of the crisis" in two French newspapers from 16 to 19 September 2008 and shows that the crisis is presented as a natural disaster, that obscures the responsible of the crisis. Then, to illustrate the second stage, the analysis studies the legitimate presentation of the crisis by the pedagogical site of the Institute for Public Financial Education (IEFP), lafinancepourtous.fr. It highlights a focus on "modest households" that both designate them as responsible and guilty.

\section{INDEX}

Keywords : economic discourse, financial literacy, IEFP, natural frame, website, governmentality Mots-clés : discours économique, éducation financière, IEFP, cadre naturel, site Internet, gouvernementalité

Code JEL A11 - Role of Economics • Role of Economists • Market for Economists, A29 - Other, D83 - Search $\bullet$ Learning $\cdot$ Information and Knowledge $\bullet$ Communication $•$ Belief $•$ Unawareness, E32 Business Fluctuations $•$ Cycles, Z13 - Economic Sociology $\bullet$ Economic Anthropology $\bullet$ Social and Economic Stratification

\section{AUTEUR}

\section{THIERRY GUILBERT}

CURAPP-ESS (UMR 7319) - UPJV 